\section{Síndrome de Desgaste Profesional manifestado en un hospital público}

Professional Wear Syndrome manifested in a public hospital

Doris Gissela Meza Menéndez

Especialista en Medicina Familiar,

Centro de Salud Junín,

abdorispeluso@hotmail.com,

https://orcid.org/0000-0002-9067-

0897, Guayaquil - Ecuador

Segundo Santiago Bueno Mejía

Especialista en Medicina Familiar,

Hospital General del Norte de

Guayaquil Los Ceibos,

santiago74bm@hotmail.com,

https://orcid.org/0000-0002-0290-

6194, Guayaquil - Ecuador

Mario Eduardo Ruíz Morocho

Médico general, Hospital General del

Norte de Guayaquil Los Ceibos,

eduardoruiz12@hotmail.com,

https://orcid.org/0000-0002-5048-

8652, Guayaquil - Ecuador

Cinthia Paola Correa Plúas

Médico general, Hospital de

especialidades Abel Gilbert Pontón,

dracp26@gmail.com,

https://orcid.org/0000-0001-8548-

4128, Guayaquil - Ecuador

http://www.jah-journal.com/index.php/jah

Journal of American health

Julio - Diciembre vol. 3. Num. 2 - 2020

Esta obra está bajo una Licencia Creative

Commons

Atribución-NoComercial-CompartirIgual

4.0 Internacional.

RECIBIDO: 20 DE MARZO 2019

ACEPTADO: 23 DE AGOSTO 2019

PUBLICADO: 2 DE JULIO 2020

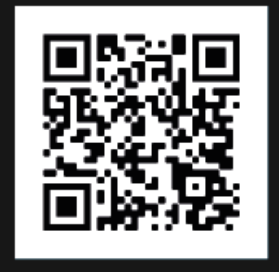

Scan this QR

code with your

smart phone or

mobile device to

read more papers

\section{RESUMEN}

Describir el nivel de desgaste laboral en una institución pública de salud ecuatoriana para que la institución considere el desarrollo de la propuesta de mejoramiento de condiciones laborales. Es un estudio descriptivo, observacional, transversal con muestreo por intención, la cuales fueron 53 enfermeras que laboran en esta entidad en el área de hospitalización y emergencia, incluye profesionales de la salud que trabajan más de dos años en la institución ambos sexos, que accedieron libre $y$ voluntariamente a participar en este trabajo de investigación. La información se recogió mediante el Instrumento Maslach Burnout Inventory, que mide cansancio emocional, despersonalización y desarrollo personal, por medio de una encuesta tomando en este caso solo se consideró las variables objeto de estudio. Observando que el nivel de agotamiento fue "medio" entre 24 y 54,5\%; según el percentil 75 y el 25 en la categoría establecida. Resultados que pueden indicar que la presentación de Burnout es directamente proporcional al tiempo de servicio transcurrido, se observa que el 9,4\% posee menos de 2 años laborando en la institución, seguidos de $3,8 \%, 13,2 \%, 3,8 \%$ con 4 años y medio de labores y los restantes $68,1 \%$ se distribuyen entre más de 5 años. La actividad en el personal de enfermería representa una condición de riesgo para la presentación de Burnout, se debe analizar las otras categorías para establecer un diagnostico final frente al desgaste laboral en esta casa de salud.

PALABRAS CLAVE: grado de desgaste, enfermería, Burnout, hospital 


\section{ABSTRACT}

Describe the level of job burnout in an Ecuadorian public health institution so that the institution considers the development of the proposal to improve working conditions. It is a descriptive, observational, cross-sectional study with sampling by intention, which was 53 nurses who work in this entity in the area of hospitalization and emergency, includes health professionals who work more than two years in the institution, both sexes, who agreed freely and voluntarily to participate in this research work. The information was collected through the Maslach Burnout Inventory Instrument, which measures emotional fatigue, depersonalization and personal development, through a survey taking in this case only the variables under study were considered. Observing that the level of exhaustion was "medium" between
24 and 54.5\%; according to the 75th and 25th percentile in the established category. Results that may indicate that the presentation of Burnout is directly proportional to the time of service elapsed, it is observed that $9.4 \%$ have less than 2 years working in the institution, followed by $3.8 \%, 13.2 \%, 3,8 \%$ with 4 and a half years of work and the remaining $68.1 \%$ are distributed among more than 5 years. The activity in the nursing staff represents a risk condition for the presentation of Burnout, the other categories must be analyzed to establish a final diagnosis against job burnout in this health house.

KEYWORDS: degree of wear, nursing, burnout, hospital

\section{INTRODUCCIÓN}

La Organización Mundial de la Salud ha procedido al reconocimiento oficial del burnout o "síndrome de estar quemado" o de desgaste profesional (1), trastorno como consecuencia de un estrés laboral crónico, y se caracteriza por un estado de agotamiento emocional, una actitud cínica o distante frente al trabajo (despersonalización), y una sensación de ineficacia y de no hacer adecuadamente las tareas. A ello se suma (2) la pérdida de habilidades para la comunicación, asimismo destaca en esta nueva clasificación del exceso de trabajo la describe como "un agotamiento físico y mental debido al estrés crónico asociado con el trabajo y el desempleo", aseguraron desde el organismo (3).

El síndrome de burnout afecta a más de la mitad de los estudiantes y profesionales involucrados en la atención médica en todo el mundo la prevalencia del SB depende del ambiente donde se desarrolle el individuo y características propias del mismo (4), el agotamiento es un síndrome psicosocial causado por condiciones de trabajo estresantes y afecta al 30-60\% del personal médico (5).

Es una realidad en la profesión de enfermería caracterizado por tres dimensiones: Agotamiento emocional se refiere al cansancio y fatiga física, psíquica o como una combinación de ambos luego la despersonalización es la segunda dimensión y se entiende como el desarrollo de sentimientos, actitudes, y respuestas negativas, distantes y frías hacia otras personas, especialmente hacia los clientes, pacientes, usuarios. Se acompaña de un incremento en la irritabilidad y una pérdida de motivación y por último el sentimiento de bajo logro surge cuando se verifica que las demandas que se le hacen exceden su capacidad para atenderlas de 
forma competente. Supone respuestas negativas hacia uno mismo y hacia su trabajo, evitación de las relaciones personales y profesionales, bajo rendimiento laboral, incapacidad para soportar la presión y una baja autoestima, como consecuencia se da la impuntualidad, la evitación del trabajo, el ausentismo y el abandono de la profesión, son síntomas habituales y típicos de esta patología laboral (6) (7).

Gil-Monte (2005) incluye un cuarto síntoma, la culpa, que permite obtener dos perfiles: en el primero, las actitudes y conductas de indolencia constituyen una estrategia de afrontamiento para los individuos, aunque tengan una connotación disfuncional para el usuario y la organización (8).

Generalmente tiende a ser más frecuente en la mujer, relacionándose con la doble carga laboral (tarea profesional y familiar), que no tienen pareja estable, La antigüedad profesional, aunque no existe un acuerdo claro de la influencia de esta variable, algunos autores han encontrado una relación positiva con el síndrome manifestada en dos períodos, correspondientes a los dos primeros años de carrera profesional y los mayores de 10 años de experiencia.

Los efectos del burnout son variados; las consecuencias fisiológicas más frecuentes son: cansancio, migraña, pérdida de peso, dolores musculares, alteraciones gastrointestinales, alteraciones respiratorias, problemas cardiovasculares, problemas del sueño e, incluso, daños a nivel cerebral (9). También existen efectos nocivos en lo psicológico, como el cinismo, la suspicacia, la agresividad, el aislamiento, la frustración y la impaciencia, que, a su vez, provocan baja calidad de vida y deterioro en las relaciones no sólo con quienes se trabaja, sino en el ámbito familiar. Incluso, el burnout da lugar a ausentismos, rotación, disminución de la productividad, fricciones entre compañeros, disminución de la calidad en el servicio, entre otras consecuencias, por lo que se afecta a la institución donde se labora (10).

Los síntomas de este síndrome son muy similares a los asociados al estrés laboral, toma nota: cambios en el estado de ánimo desmotivación, agotamiento mental, sensibilidad a la crítica, falta de energía y menor rendimiento, afecciones del sistema locomotor, dolor y rigidez muscular, problemas gastrointestinales, problemas cardiovasculares, alteraciones en la piel, mareos, Alteraciones del apetito sexual, Obesidad (11).

EL propósito es describir el grado de desgaste en enfermeros que padecen síndrome de burnout para el desarrollo de la propuesta de mejoramiento de condiciones laborales del personal del hospital público de Ecuador.

\section{MATERIALES Y MÉTODOS}

Estudio descriptivo, observacional de corte transversal, con muestreo por intención, realizado en una muestra de 53 enfermeras, de un Hospital público en Ecuador; que firmaron el consentimiento informado para participar en el estudio de forma voluntaria, a través de encuesta electrónica anónima por correo electrónico en Google Drive, aplicamos un cuestionario estructurado desarrollado por los autores sobre datos sociodemográficos, y tomamos los datos objeto de estudio como es el grado de desgaste laboral. 
La recolección de los datos se hizo mediante el instrumento Maslach Burnout Inventory (MBI); propuesto por Maslach y Yackson (1986), que plantea una serie de preguntas relacionadas a los sentimientos y pensamientos con relación a su interacción laboral; instrumento estructurado por 22 ítems, tipo Likert, con valores que oscilan en un rango de 0 a 6, que van desde el criterio 'nunca' a 'diariamente'; los 22 ítems conforman las siguientes subescalas: Cansancio emocional (CE), despersonalización (DP) y realización personal (RP). El incremento de puntuación en (CE y (DP), es indicativo de Burnout alto, opuesto a lo que refleja la escala de (RP). Sin embargo solo se tomó (CE) para análisis en esta investigación. El análisis estadístico se realizó con la medida de frecuencias la que permitió valorar el grado de Burnout en la escala emocional, además se relacionó el síndrome de Burnout, con las variables edad y tiempo de vinculación. El grupo de variables que aluden cansancio emocional están representadas por puntuaciones altas y bajas; Los Resultados por encima del percentil 75 se incluyen en la categoría "alto", entre el percentil 75 y el 25 en la categoría "medio" y, por debajo del percentil 25, en la categoría "bajo".

\section{RESULTADOS}

Se detalla el grado de agotamiento por parte de las enfermeras que laboran en una institución pública del ecuador

Tabla \# 1: Distribución de enfermeros que realizaron la encuetas por sexo

\begin{tabular}{|c|c|c|}
\hline Sexo & Frecuencia & $\%$ \\
\hline Femenino & 42 & 79,2 \\
\hline Masculino & 11 & 20,7 \\
\hline $\begin{array}{c}\text { Total } \\
\text { general }\end{array}$ & 53 & $100 \%$ \\
\hline
\end{tabular}

Tomado de encuesta virtual realizada a las enfermeras del hospital

Según el sexo el 79,25 del personal de salud corresponde al género femenino y el 2,7 \% corresponde al sexo masculino.

Tabla \# 2 Distribución de agotamiento laboral

\begin{tabular}{|c|c|c|}
\hline Diariamente & $\mathbf{2}$ & $\mathbf{3 , 7}$ \\
\hline Nunca & 10 & 18,8 \\
\hline Pocas Veces A La Semana & 4 & 7,5 \\
\hline Pocas Veces Al Año O Menos & 14 & 26,4 \\
\hline Una Vez A La Semana & 4 & 7,5 \\
\hline Una Vez Al Mes O Menos & 5 & 9,4 \\
\hline Unas Pocas Veces Al Mes & 14 & 26,4 \\
\hline Total general & 53 & 100 \\
\hline
\end{tabular}

Tomado de encuesta virtual realizada a las enfermeras del hospital

En cuanto al grado de agotamiento el 18,8 \% refiere nunca sentir agotamiento, el 26,4\% refiere sentir algunas pocas veces al año. Por otro lado, el 9,4 \% una vez al mes, 7,5 \% una vez a la 
semana, $7,5 \%$ pocas veces a la semana y 3,7\% diariamente, (siendo el nivel de agotamiento medio con un $54,5 \%$. Según el percentil 75 y el 25 en la categoría corresponde a nivel medio).

Tabla \# 3 Frecuencia según escala de Likert por grado de Agotamiento en el trabajo, según trabajar todo el día con la gente me cansa

\begin{tabular}{|c|c|c|}
\hline Diariamente & $\mathbf{5}$ & $\mathbf{9 , 4}$ \\
\hline Nunca & 11 & 20,7 \\
\hline Pocas Veces A La Semana & 3 & 5,6 \\
\hline Pocas Veces Al Año O Menos & 26 & 49,0 \\
\hline Una Vez A La Semana & 3 & 5,6 \\
\hline Una Vez Al Mes O Menos & 4 & 7,5 \\
\hline Unas Pocas Veces Al Mes & 1 & 1,8 \\
\hline Total general & $\mathbf{5 3}$ & 100 \\
\hline
\end{tabular}

Esta tabla se observa la relación entre datos observados en los resultados previos donde el 20 $\%$ de los trabajadores de salud indica nunca sentirse agotado seguidos el $49,0 \%$ pocas veces al año, sin embargo el $9 \%$ siente agotamiento diariamente, seguido de 7,5\%, 5,6\%, 5,6\%, 1,8\%, siendo el nivel de agotamiento medio con un $29,9 \%$. Recordemos que para considerar este nivel debe obtenerse resultados entre el percentil 75 y el 25 en la categoría.

Tabla \# 4 Frecuencia de enfermeras que siente desgaste físico y mental

\begin{tabular}{|c|c|c|}
\hline Diariamente & 39 & 73,58490566 \\
\hline $\begin{array}{c}\text { Nunca } \\
\begin{array}{c}\text { Pocas Veces A La } \\
\text { Semana }\end{array}\end{array}$ & 2 & 3,773584906 \\
\hline $\begin{array}{c}\text { Pocas Veces Al } \\
\text { Año O Menos }\end{array}$ & 6 & 11,547169811 \\
\hline $\begin{array}{c}\text { Una Vez A La } \\
\text { Semana }\end{array}$ & 1 & 1,886792453 \\
\hline $\begin{array}{c}\text { Unas Pocas } \\
\text { Veces Al Mes }\end{array}$ & 1 & 1,886792453 \\
\hline Total general & 53 & 100 \\
\hline
\end{tabular}

Tomado de encuesta virtual realizada a las enfermeras del hospital

Tabla \# 5 enfermeros que sienten más duro con la gente

\begin{tabular}{|c|c|c|c|c|c|}
\hline Nunca & $\begin{array}{l}\text { Pocas } \\
\text { veces-año }\end{array}$ & $\begin{array}{l}\text { Una vez } \\
\text { al mes }\end{array}$ & $\begin{array}{l}\text { Una vez a } \\
\text { la semana }\end{array}$ & $\begin{array}{l}\text { Pocas } \\
\text { veces a la } \\
\text { semana }\end{array}$ & $\begin{array}{l}\text { Diaria } \\
\text { ment } \\
\text { e }\end{array}$ \\
\hline $37 \%$ & $37, / \%$ & $5,7 \%$ & $1,9 \%$ & $13,2 \%$ & $3,2 \%$ \\
\hline
\end{tabular}

Tomado de encuesta virtual realizada a las enfermeras del hospital 
Respecto a las emociones que manejan los enfermeros sobre los pacientes, el $37 \%$ refiere que nunca ha tenidos la necesidad de ser enérgico con los pacientes seguidos de $37,7 \%$. Sin embargo, el $13,2 \%, 5,7$ y 1,9\%, siendo el nivel de agotamiento bajo con un $24 \%$; para considerar este nivel debe obtenerse resultados del percentil menor de 25 en la categoría.

\section{Grafico \# 1 intereses de la institución sobre sus problemas emocionales}

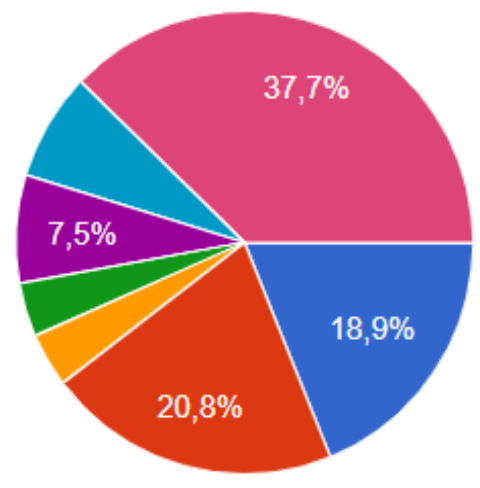

NUNCA

pocas veces al año o menos

UNA VEZ AL MES O MENOS

UNAS POCAS VECES AL MES

UNA VEZ A LA SEMANA

pocas Veces a la semana

DIARIAMENTE

Tomado de encuesta virtual realizada a las enfermeras del hospital

En relación a las soluciones que brinda la institución a este grupo entre el 185 y $20 \%$, siente que son abordados de forma frecuente sus problemas emocionales, sin emabrgo el $37.7 \%, 20 \%$ , 18,9\%, 13,2, 9,4\% 7,5\%, 5,7 \% refieren que no son tratados de forma adecuadamente frente a sus emociones.

\section{Grafico \# 2 Distribución de años laborados en la institución}

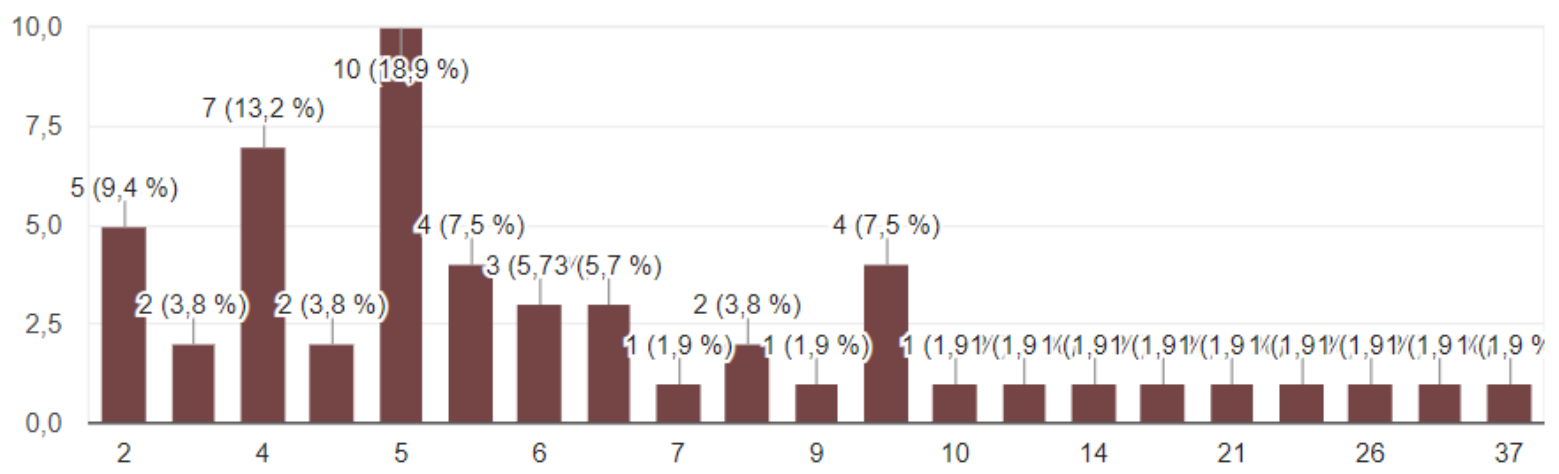

Tomado de encuesta virtual realizada a las enfermeras del hospital

Se observa que el 9,4\% posee menos de 2 años laborando en la institución, seguidos de 3,8\%, $13,2 \%, 3,8 \%$; 4 años y medio de labores y los restantes $68,1 \%$ se distribuyen entre más de 5 años.

\section{DISCUSIÓN}

El síndrome de desgaste o burnout suele presentarse con mayor frecuencia en aquellos puestos de trabajo relacionados con atención a terceros, como: docentes, personal sanitario o personas 
que trabajan en atención al cliente, de hecho un estudio multicentrico de catorce trabajos en enfermeras en el área de ginecología por De la Fuente-Solana et al, 2019 señala una $n=464$ enfermeras, donde se observó prevalencias según las variables agotamiento emocional $29 \%$ (IC 95\%: 11-52\%). Las variables de agotamiento consideradas fueron sociodemográficas (edad, estado civil, número de hijos, sexo), relacionadas con el trabajo (duración de la jornada laboral, relación enfermera-paciente, experiencia), presentando altos niveles de burnout síndrome (12) , lo que difiere con los resultados de esta investigacion donde el grado de agotamiento laboral corresponde al nivel medio.

En un estudio elaborado por Ugalde, 2018, señala que el $77.3 \%$ de personal encuestado tiene un grado de afectación importante en la dimensión de cansancio emocional, siendo el grupo vulnerable el sexo masculino casados que actúan en horario diurno y que su edad fluctúa entre 30 y 40 años, (13), lo que difiere con los resultados, siendo el sexo masculino el mas afectado asi como en frecuencia mucho menor en esta escala. Por otro lado Gutierez Lemes , 2016 en su trabajo titulado Prevalencia del síndrome de Burnout en profesionales de enfermería de Orinoquia, Colombia en 2016 con 100 profesionales de enfermería se observó que el $90 \%$ de la muestra estudiada eran mujeres, $53 \%$ solteras, $54 \%$ menores de 28 años y $42 \%$ sin hijos. La prevalencia general del síndrome de Burnout fue del 16\%, en el área de atención hospitalaria fue del $25,5 \%$ y en áreas distintas a esta fue del $6,1 \%$. En el área hospitalaria, hubo mayores proporciones en fatiga emocional con un 42\%, Ser menor de 28 años se asoció con una mayor prevalencia. (14). Víctor Ramón Miranda-Lara, 2016 denominado prevalencia del síndrome de burnout en personal de enfermería de dos instituciones en la ciudad de Mexico donde aplicaron una encuesta en 535 señala que la proporción de personal con síndrome de burnout fue de $33.8 \%$ (181 de 535), el $6.7 \%$ (36 de 535) presentó un alto nivel de este síndrome. Se encontró que $44.1 \%$ presentó agotamiento emocional cifras similares a las expuestas este trabajo, si consideramos el nivel de afectación medio. (15).

\section{CONCLUSIONES}

El grado de agotamiento por parte de las enfermeras en esta institución es medio encontrándose entre el percentil 75 y el 25 en la categoría; de mayor proporción en sexo femenino, es directamente proporcional al tiempo de servicio transcurrido con una media de 5 años con el $18 \%$ y $68 \%$ de ellos con más de 5 años de antigüedad.

No existe un consenso específico sobre cuáles son las estrategias más apropiadas para enfrentar el problema de agotamiento en profesionales de la salud. Algunos trabajos refieren que la experiencia de los educadores médicos y de salud es crucial para el diseño de estrategias efectivas para abordar este problema.

Por lo que debemos diseñar programas de prevención encaminados a modificar las conductas y atributos que mejoren el ambiente laboral y que sea aplicable y sostenido.

\section{REFERENCIAS}


1. OMC. (28 de Mayo de 2019). Recuperado el 5 de Diciembre de 2019, de Medico paciente : http://www.medicosypacientes.com/articulo/la-oms-reconoce-comoenfermedad-el-burnout-o-sindrome-de-estar-quemado

2. Bitran M, Z. D. (Abril de 2019). Burnout en estudiantes de profesiones sanitarias. Factores de riesgo y protección. Rev Med Chil, 147(4), 10-517.

3. Infobase. (28 de Mayo de 2019). Infobase. Obtenido de https://www.infobae.com/salud/2019/05/28/para-la-oms-el-sindrome-de-burnoutes-un-trastorno-mental/

4. Lachiner Saborío Morales, Med. leg. Costa Rica vol.32 n.1 Heredia Jan./Mar. 2015

5. Maria Rodríguez. Síndrome de burnout en especialistas de medicina interna y factores asociados a su desarrollo revista clínica española · diciembre 2019

6. Velando-Soriano A 1, O.-C. E.-U.-B.-D. (Enero de 2020). Impacto del apoyo social en la prevención del síndrome de burnout en enfermeras: una revisión sistemática. Jpn J Nurs Sci, 17(1), e12269. doi: 10.1111 / jjns.12269.

7. Apiquian Guitart, A. (26 de Abril de 2007). Obtenido de Tercer Congreso de Escuelas de Psicología de las Universidades Red Anáhuac.: https://www.ucm.es/data/cont/media/www/pag-30022/sindrome\%20burnout.pd

8. Gil-Monte, P. R. (2003). El síndrome de quemarse or el trabajo en orfesionales de enfereria. Revista Eletrônica InterAção Psy, 1, 19-33.

9. Kahill, S. (1988). Síntomas del agotamiento profesional: una revisión de la evidencia empírica. Canadian Psychology / Psychologie canadienne, 29 (3), 284297. https://doi.org/10.1037/h0079772

10. Juárez-García, A. I.-Á.-R. (2014). Síndrome de burnout en población mexicana: Una revisión sistemática. a. Salud Mental, 39, 159, 176.

11. Escobar, P. (16 de Mayo de 2016). El síndrome de burnout también llamado "síndrome del trabajador quemado" está relacionado con una respuesta de estrés crónico en el trabajo. Neurociencias de Junta Beneficiencia, $s / d$.

12. De la Fuente-Solana EI, S.-M. N.-H.-U.-D.-G. (19 de Julio de 2019). Prevalencia, factores relacionados y niveles del síndrome de burnout entre las enfermeras que trabajan en servicios de ginecología y obstetricia: una revisión sistemática y un metanálisis. Int J Environ Res Salud Pública, 16(14), E2585. doi: 10.3390 / ijerph16142585.

13. UGALDE Vicuña, M. M. (Diciembre de 2018). Burnout en docentes de la Facultad de Ingeniería Industrial de. Espacios.

14. Gutierez Lemes, Prevalencia del síndrome de Burnout en profesionales de enfermería de Orinoquia, Colombia en 2016, Univ. Salud [en línea]. 2018, vol.20, n.1, pp.37-43. ISSN 0124-7107:http://www.scielo.org.co/scielo.php?pid=S012471072018000100037\&script=sci_abstract\&tlng=en

15. Miranda-Lara VR, Monzalvo-Herrera G, Hernández-Caballero B, Ocampo-Torres $M$. Prevalencia del síndrome de burnout en personal de enfermería de dos instituciones de salud. Rev Enferm Inst Mex Seguro Soc. 2016;24(2):115-119 